www. revistadyo.com

\title{
Ellas, líderes: un estudio del contexto empresarial iberoamericano
}

Irene Campos-García

Recibido: 6 de Febrero de 2021

Aceptado: 5 de Abril de 2021

https://doi.org/10.37610/dyo.v0i74.604

\section{Resumen}

Pese al incremento de las mujeres en posiciones de liderazgo, aún existen barreras que dificultan su acceso y visibilidad como miembros top de las organizaciones. Este trabajo pretende evidenciar la infrarrepresentación de las líderes en el contexto empresarial iberoamericano y examinar las iniciativas que las compañías que allí operan han implantado a fin de impulsar el liderazgo femenino. Para ello, a partir del ranking MERCO Líderes y los informes corporativos de sostenibilidad y/o diversidad de cada compañía, se realiza un análisis tanto cuantitativo como cualitativo durante el periodo 2013-2020. De las conclusiones obtenidas se derivan ciertas reflexiones que implican a autoridades gubernamentales, compañías y la sociedad en su conjunto.

\section{Palabras clave}

Representación femenina, mujeres líderes, posiciones de liderazgo, Iberoamérica.

\section{Introducción}

En las últimas décadas se ha producido un incremento de la representación femenina en el mercado laboral — actualmente, la tasa de participación de las mujeres en la fuerza laboral mundial es del 47\% (The World Bank, 2020). A pesar de que casi la mitad de la población femenina está empleada y sus tasas de participación van gradualmente acercándose a las de los hombres en muchos países desarrollados y emergentes, la brecha de género en el mercado laboral está muy latente, sobre todo en lo que se refiere al acceso y promoción a las posiciones de liderazgo. Ellas ocupan sólo algo más de una cuarta parte (29\%) de los cargos directivos en todo el mundo (Catalyst, 2020) y son aún más minoritarias como números uno de las organizaciones. La proporción de directoras ejecutivas es muy baja: sólo 37 empresas del listado Fortune 500 están lideradas por mujeres, lo que supone un $7,4 \%$ (Fortune, 2020).

Este desequilibrio de género ha colocado a las mujeres en el centro del debate social y político en muchos países. La literatura académica y los medios de comunicación han prestado una mayor atención a las féminas, dando ello lugar, en los últimos años, al uso extendido de términos como 'techo de cristal' o 'acantilado de cristal' para describir las numerosas barreras a las que deben enfrentarse en los lugares de trabajo. También muchos países han establecido la necesidad de implantar sistemas de cuotas, guías o códigos de buen gobierno corporativo para lograr una mayor representación femenina en posiciones de liderazgo

\footnotetext{
$凶 \quad$ Irene Campos García * irene.campos@urjc.es

* Universidad Rey Juan Carlos

Economía de la Empresa (ADO), Economía Aplicada II

y Fundamentos del Análisis Económico

Paseo de los Artilleros, s/n

28032 Madrid (España)
}

(Eriksson-Zetterquist y Styhre, 2008; Deloitte, 2019; Olsen, Parsons, Martins y Ivanaj, 2016). Tales medidas no sólo se justifican por razones éticas y de igualdad, sino también por razones de eficiencia económica, ya que numerosas investigaciones han enfatizado las ventajas del liderazgo femenino, insistiendo en los beneficios y valores que las mujeres aportan a las organizaciones (p.ej., Eagly y Carli, 2003; Helgesen, 1995; Rosener, 2011). La Teoría de los Roles Sociales ha explicado por qué hombres y mujeres se comportan de manera diferente: los hombres son generalmente más asertivos, competitivos y dominantes, mientras que las mujeres son más cooperativas y empáticas, se muestran más desinteresadas, altruistas, transparentes y preocupadas por los demás y tienden a ser más aversas al riesgo (p.ej., Eagly y Wood, 2011; Rosener, 2011). De ahí la importancia de la diversidad e inclusión y los beneficios de aprovechar y explotar el talento femenino (Swailes, Downs y Orr, 2014).

El objetivo de este trabajo se concreta en analizar la evolución de las mujeres líderes en el panorama empresarial iberoamericano y explorar las prácticas o iniciativas que sus compañías han puesto en marcha para visibilizar, empoderar y explotar el talento femenino. Por tanto, este estudio se realiza en dos etapas. En la primera de ellas, donde se lleva a cabo un análisis descriptivo cuantitativo, se examina el ranking MERCO Líderes en España y Latinoamérica durante 2013-2020 -MERCO publica este ranking en cada país con el objetivo de identificar a sus 100 líderes empresariales más influyentes o reconocidos. En la segunda etapa se realiza un análisis cualitativo que permite comparar las prácticas en pro del fomento del liderazgo femenino que las compañías transnacionales y multinacionales para las que trabajan las mujeres incluidas en el ranking han implantado y desarrollado durante el periodo seleccionado. Dicho análisis se lleva a cabo a partir de la información contenida en las Memorias Anuales, los Informes de Sostenibilidad/ Diversidad e Inclusión y/o los sitios web corporativos. 
Este trabajo contribuye, por tanto, a un mayor conocimiento y comprensión de la situación actual en el panorama empresarial iberoamericano y de los esfuerzos que se están o no llevando a cabo en lo que a promoción de mujeres se refiere. Tales conclusiones permiten, por un lado, identificar los principales retos que se plantean en materia de género en el ámbito empresarial y social de ambas geografías y, por otro, sugerir algunas líneas de acción que implican a autoridades gubernamentales, compañías y la sociedad en su conjunto.

El trabajo se estructura como sigue. La segunda sección describe el método y detalla la muestra y el procedimiento. La tercera sección muestra la evolución de la visibilidad femenina en el Top 100 del ranking MERCO Líderes en España y países latinoamericanos durante el periodo 20132020 y analiza comparativamente las acciones e iniciativas puestas en marcha por las compañías para potenciar el liderazgo femenino. A continuación se discuten los resultados obtenidos $\mathrm{y}$, finalmente, se presentan las conclusiones e implicaciones del trabajo.

\section{Método}

En primer lugar, con el objetivo de analizar la evolución y evidenciar la infrarrepresentación de las mujeres líderes en el panorama empresarial iberoamericano se procedió a la recopilación de los listados del ranking MERCO Líderes en cada país a partir de 2013. Aunque este ranking lleva publicándose en España desde 2001, en Colombia desde 2008 o en Argentina y Chile desde 2010, no es hasta 2013 cuando aparecen los listados en la mayoría de países latinoamericanos -concretamente, en Ecuador, Bolivia, Brasil, México y Perú. No obstante, el ranking para Costa Rica, Panamá y Uruguay está disponible a partir de 2017, 2018 y 2019, respectivamente. De ahí que la elección de la longitud del estudio atienda al periodo comprendido entre 2013 y 2020 para el que existen más datos disponibles. Seguidamente se llevó a cabo el examen y recuento de las líderes femeninas incluidas en el listado anual de cada país, así como la identificación de las compañías para las que trabajan.
En segundo lugar, se procedió a la búsqueda y colección de la información y variables relacionadas con la diversidad -indicadores GRI de rendimiento social - y las iniciativas dirigidas al fomento del liderazgo femenino a partir de los Informes Anuales Corporativos, las Memorias Anuales de Sostenibilidad y/o Diversidad y los sitios web corporativos de cada compañía. Durante esta etapa se evidenciaron tres cuestiones que merecen ser destacadas: 1) aspectos relativos a la diversidad y equidad de género comienzan a acaparar más atención en las publicaciones a partir de 2016-2017; 2) existen importantes diferencias en la relevancia que las empresas multinacionales otorgan a la estrategia y políticas de diversidad frente a las empresas nacionales — de ahí que la mayoría de compañías de menor tamaño y/o que operan en un ámbito estrictamente nacional no ofrezcan información al respecto; y 3) se denota una falta de homogeneidad en las variables y medidas sobre diversidad publicadas por las empresas. Ante tales limitaciones, este trabajo incluye en el análisis a aquellas compañías transnacionales y/o multinacionales que han conseguido a lo largo del periodo posicionarse en el Top 100 en más de un país iberoamericano - es el caso de Microsoft, Siemens, McDonald's, Santander, Manpower, Telefónica, Coca Cola, Facebook, Google, General Electric, P\&G, Pepsico, Latam Airlines, Kimberly Clark, Unilever, Nestlé, Yanbal, Petrobras, Walmart y DHL. Estas compañías son, además, las que en general muestran una mayor o más completa información acerca de las políticas y acciones en pro del liderazgo femenino y la igualdad de género desde 2013, lo que facilita el análisis para describir y comparar cualitativamente las acciones encaminadas a visibilizar, empoderar y explotar el talento femenino.

\section{Resultados}

\section{Resultados del análisis cuantitativo}

A partir del examen del ranking MERCO Líderes en cada país durante 2013-2020, la Tabla 1 muestra el número de mujeres que, por su influencia o reconocimiento, han aparecido en el Top 100.

Tabla 1 Número de

mujeres en el Top 100

Fuente: Elaboración propia

a partir del ranking

MERCO Líderes

*Sólo 50 posiciones

incluidas en el ranking en

2018 y 75 en 2019

\begin{tabular}{lcccccccc}
\hline \multicolumn{1}{c}{ País } & $\mathbf{2 0 1 3}$ & $\mathbf{2 0 1 4}$ & $\mathbf{2 0 1 5}$ & $\mathbf{2 0 1 6}$ & $\mathbf{2 0 1 7}$ & $\mathbf{2 0 1 8}$ & $\mathbf{2 0 1 9}$ & $\mathbf{2 0 2 0}$ \\
\hline España & 16 & 19 & 17 & 17 & 19 & 21 & 22 & 22 \\
\hline Colombia & 6 & 8 & 7 & 9 & 9 & 8 & 10 & 12 \\
\hline Argentina & 3 & 2 & 3 & 4 & 4 & 4 & 7 & 10 \\
\hline Chile & 5 & 2 & 2 & 2 & 6 & 6 & 7 & 7 \\
\hline Ecuador & 8 & 10 & 13 & - & 13 & 14 & 17 & - \\
\hline Bolivia & 9 & 11 & 9 & 9 & 12 & 10 & 11 & - \\
\hline Brasil & 7 & 5 & - & 3 & 7 & 9 & 10 & - \\
\hline México & 9 & 5 & 2 & 3 & 7 & 11 & 11 & 16 \\
\hline Perú & 7 & 5 & 7 & 8 & 11 & 14 & 16 & 21 \\
\hline Costa Rica & - & - & - & - & 12 & 17 & 14 & 19 \\
\hline Panamá* & - & - & - & - & - & 9 & 12 & - \\
\hline Uruguay & - & - & - & - & - & - & 20 & 18 \\
\hline
\end{tabular}


A partir de los datos disponibles, puede comprobarse que España, Perú, Costa Rica y Uruguay son los países que, al final del periodo, cuentan con un mayor número de mujeres reconocidas por su papel de líder. Por el contrario, el menor número de mujeres se encuentra en el ranking de Chile que, al igual que el de Bolivia y Brasil, da muestras de un escaso avance en cuanto a visibilidad femenina se refiere. También en Argentina sólo el $10 \%$ de los puestos está ocupado por mujeres pero, a diferencia de Chile, consigue triplicar sus cifras durante el periodo, al igual que Perú. Por su parte, Ecuador y Colombia (aunque este último más rezagado) consiguen duplicar la presencia femenina en el ranking.

De manera específica, España ha mostrado una ligera tendencia positiva, estando ésta marcada por el estancamiento de las cifras durante el periodo. En 2015 ellas comienzan a adelantar posiciones en el Top 20 y en 2017 una decena de mujeres forma parte del Top 30. Aunque en los dos últimos años se produce un ligero retroceso en los puestos, 13 de las 22 líderes se posicionan en 2020 en el Top 50.

En el ranking colombiano, durante 2014 y 2015 aparece una mujer en el Top 20 y dos de ellas en 2018. La visibilidad femenina mejora en 2019, con 4 mujeres en el Top 30. Finalmente, en 2020 se suman más mujeres al listado, estando 4 de ellas incluidas en el Top 50.

Argentina consigue en 2016 colocar a una mujer en el Top 10, comenzando todas ellas a adelantar puestos en 2018. Los años 2019 y 2020 suponen el avance más significativo en número, siendo 3 y 4 las mujeres, respectivamente, las que ocupan posiciones dentro del Top 50 .

En Chile, las 10 primeras posiciones apenas varían hasta 2016, estando ocupadas por los mismos líderes masculinos y permaneciendo las mujeres en la cola del ranking hasta que, en 2017, comienzan a escalar puestos e incrementan su presencia notablemente. No existe apenas avance en cuanto al número de mujeres al final del periodo pero en 20204 de ellas (de un total de 7) forman parte del Top 50.

Por lo que respecta a Ecuador, desde 2013 hasta 2018 una mujer encabeza el listado y las féminas adelantan posiciones a partir de 2017, situándose 4 en el Top 20. Al final del periodo, 11 de 17 ocupan posiciones en la primera mitad de la clasificación.
El ranking boliviano muestra pocas variaciones en cuanto a cifras, aunque las mujeres comienzan a escalar puestos a partir de 2017, habiendo 6 de ellas en las 50 primeras posiciones en 2019.

Brasil posiciona, a lo largo de todo el periodo, a una mujer en el primer puesto del ranking, aunque en general refleja poco avance de las líderes en lo que a número y escaladas se refiere. Aun así, 6 de las 10 líderes se colocan en 2019 en el Top 50.

En México las mujeres comienzan a crecer en número y a avanzar posiciones a partir de 2017 , siendo una mujer en 2018 y 2019 la segunda líder mejor valorada. Doblando casi la representación al final del periodo, 6 mujeres (de un total de 16) ocupan el Top 50.

En el listado peruano siempre aparecen durante los primeros años los mismos líderes masculinos en los primeros puestos, empezando las mujeres a ascender posiciones y a tener una mayor representatividad a partir de 2016. De un total de 21 mujeres, 8 de ellas se posicionan en 2020 en la primera mitad del ranking.

Costa Rica estrena el ranking en 2017 con un total de 12 y hasta el final del periodo suma 7 más. Todos los años son 9 las mujeres que se posicionan en el Top 50.

El ranking panameño, que comienza a publicarse en 2018, sólo incorpora 50 posiciones, estando 9 mujeres incluidas en él. En 2019 el listado se amplía a 75 posiciones, siendo 8 las mujeres que forman parte del Top 50.

Por último, Uruguay cuenta en 2019 con un total de 20 féminas, estando 13 de ellas en las primeras cincuenta posiciones y 5 en el Top 20. Sin embargo, en 2020 reduce ligeramente la representación femenina, descendiendo puestos también la mayoría de mujeres - 4 y 9 en el Top 20 y Top 50, respectivamente.

\section{Resultados del análisis cualitativo}

La Tabla 2 recoge, por países, el listado de compañías cuyas líderes han aparecido en el ranking MERCO Líderes durante el periodo seleccionado . 
Tabla 2 Empresas reconocidas por sus líderes femeninas.

Fuente: $\quad$ Elaboración propia a partir de MERCO Lideres

\begin{tabular}{|c|c|c|c|c|c|c|c|c|}
\hline $\begin{array}{l}\text { Año } \\
\text { País }\end{array}$ & 2013 & 2014 & 2015 & 2016 & 2017 & 2018 & 2019 & 2020 \\
\hline : & $\begin{array}{l}\text { Microsoft } \\
\text { Bankinter } \\
\text { Siemens } \\
\text { McDonald's } \\
\text { Codorniú } \\
\text { Día } \\
\text { Amazon } \\
\text { ING } \\
\text { FCC } \\
\text { Santander } \\
\text { Unicef } \\
\text { ESADE } \\
\text { Ikea } \\
\text { Manpower } \\
\text { Iberdrola } \\
\text { Telefónica }\end{array}$ & $\begin{array}{l}\text { Bankinter } \\
\text { Día } \\
\text { Siemens } \\
\text { Microsoft } \\
\text { FCC } \\
\text { Ikea } \\
\text { Santander } \\
\text { Codorniú } \\
\text { McDonald's } \\
\text { Prosegur } \\
\text { Amazon } \\
\text { ING } \\
\text { ESADE } \\
\text { Entradas.com } \\
\text { Unicef } \\
\text { FCC } \\
\text { IBM } \\
\text { CocaCola } \\
\text { Telefónica }\end{array}$ & $\begin{array}{l}\text { Santander } \\
\text { Bankinter } \\
\text { Siemens } \\
\text { Día } \\
\text { Microsoft } \\
\text { Ikea } \\
\text { Codorniú } \\
\text { FCC } \\
\text { Prosegur } \\
\text { Inditex } \\
\text { IBM } \\
\text { Unicef } \\
\text { McDonald's } \\
\text { Amazon } \\
\text { ING } \\
\text { ESADE }\end{array}$ & $\begin{array}{l}\text { Santander } \\
\text { Bankinter } \\
\text { Siemens } \\
\text { Día } \\
\text { Microsoft } \\
\text { Ikea } \\
\text { Codorniú } \\
\text { IBM } \\
\text { Inditex } \\
\text { ING } \\
\text { FCC } \\
\text { Prosegur } \\
\text { CocaCola } \\
\text { McDonald's } \\
\text { Amazon } \\
\text { ESADE }\end{array}$ & $\begin{array}{l}\text { Santander } \\
\text { Bankinter } \\
\text { Siemens } \\
\text { Día } \\
\text { Ikea } \\
\text { Inditex } \\
\text { Microsoft } \\
\text { Amazon } \\
\text { CocaCola } \\
\text { ING } \\
\text { ESADE } \\
\text { IBM } \\
\text { FCC } \\
\text { Prosegur } \\
\text { Codorniú } \\
\text { McDonald's } \\
\text { Facebook } \\
\text { Google }\end{array}$ & $\begin{array}{l}\text { Santander } \\
\text { Bankinter } \\
\text { Siemens } \\
\text { Google } \\
\text { Ikea } \\
\text { Amazon } \\
\text { Día } \\
\text { Microsoft } \\
\text { Inditex } \\
\text { CocaCola } \\
\text { ING } \\
\text { ESADE } \\
\text { IBM } \\
\text { Prosegur } \\
\text { Codorniú } \\
\text { FCC } \\
\text { Facebook } \\
\text { McDonald's } \\
\text { Holaluz } \\
\text { FritRavich }\end{array}$ & $\begin{array}{l}\text { Santander } \\
\text { Bankinter } \\
\text { Google } \\
\text { Ikea } \\
\text { CocaCola } \\
\text { Microsoft } \\
\text { IBM } \\
\text { Inditex } \\
\text { ING } \\
\text { Iberdrola } \\
\text { FCC } \\
\text { Prosegur } \\
\text { LinkedIn } \\
\text { HP } \\
\text { Facebook } \\
\text { McDonald's } \\
\text { FritRavich } \\
\text { Holaluz } \\
\text { ESADE } \\
\text { Codorniú }\end{array}$ & $\begin{array}{l}\text { Santander } \\
\text { Bankinter } \\
\text { Ikea } \\
\text { Google } \\
\text { CocaCola } \\
\text { Microsoft } \\
\text { Adobe } \\
\text { Inditex } \\
\text { ING } \\
\text { IBM } \\
\text { Iberdrola } \\
\text { Holaluz } \\
\text { HP } \\
\text { ElCorteInglés } \\
\text { ESADE } \\
\text { Prosegur } \\
\text { McDonald's } \\
\text { Facebook } \\
\text { FCC } \\
\text { Habitissimo } \\
\text { ASTI }\end{array}$ \\
\hline 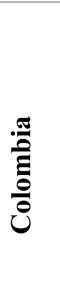 & $\begin{array}{l}\text { Servientrega } \\
\text { Terpel } \\
\text { G.Nutresa } \\
\text { Cám.Comercio } \\
\text { Helm Andina } \\
\text { P\&G }\end{array}$ & $\begin{array}{l}\text { Servientrega } \\
\text { Terpel } \\
\text { G.Nutresa } \\
\text { P\&G } \\
\text { Bancamía } \\
\text { Pepsico } \\
\text { Cám.Comercio } \\
\text { Energ,Bogotá }\end{array}$ & $\begin{array}{l}\text { Terpel } \\
\text { Procolombia } \\
\text { Servientrega } \\
\text { Asobancaria } \\
\text { G.Nutresa } \\
\text { Cám.Comercio } \\
\text { P\&G }\end{array}$ & $\begin{array}{l}\text { Terpel } \\
\text { M.Medellín } \\
\text { G.Nutresa } \\
\text { Cám.Comercio } \\
\text { Google } \\
\text { Servientrega } \\
\text { Equion } \\
\text { Procolombia } \\
\text { Pepsico }\end{array}$ & $\begin{array}{l}\text { Terpel } \\
\text { G.Nutresa } \\
\text { Google } \\
\text { Crepes\&Waffles } \\
\text { Cám.Comercio } \\
\text { Servientrega } \\
\text { MetroMedellín } \\
\text { Pepsico } \\
\text { Equion }\end{array}$ & $\begin{array}{l}\text { Terpel } \\
\text { G.Nutresa } \\
\text { Crepes\&Waffles } \\
\text { Cám.Comercio } \\
\text { DiscoveryNetw. } \\
\text { Pepsico } \\
\text { Equion } \\
\text { Energ.Bogotá }\end{array}$ & $\begin{array}{l}\text { Terpel } \\
\text { Crepes\&Waffles } \\
\text { G.Nutresa } \\
\text { Procafecol } \\
\text { UNColombia } \\
\text { Cám.Comercio } \\
\text { DiscoveryNetw. } \\
\text { Equion } \\
\text { Pepsico } \\
\text { Energ.Bogotá }\end{array}$ & $\begin{array}{l}\text { Crepes\&Waffles } \\
\text { Terpel } \\
\text { Procafecol } \\
\text { G.Nutresa } \\
\text { UNColombia } \\
\text { Suramericana } \\
\text { Univ.EAN } \\
\text { Pepsico } \\
\text { Energ.Bogotá } \\
\text { Corficolombiana } \\
\text { DiscoveryNetw. } \\
\text { Proantioquía }\end{array}$ \\
\hline 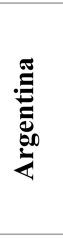 & $\begin{array}{l}\text { Google } \\
\text { Microsoft } \\
\text { G.Motors }\end{array}$ & $\begin{array}{l}\text { G.Motors } \\
\text { Google }\end{array}$ & $\begin{array}{l}\text { G.Motors } \\
\text { Microsoft } \\
\text { Google }\end{array}$ & $\begin{array}{l}\text { Aer.Argentinas } \\
\text { Microsoft } \\
\text { Google } \\
\text { LatamAirl. }\end{array}$ & $\begin{array}{l}\text { Google } \\
\text { GrupoST } \\
\text { LatamAirl. } \\
\text { Microsoft }\end{array}$ & $\begin{array}{l}\text { GrupoST } \\
\text { Google } \\
\text { Microsoft } \\
\text { LatamAirl. }\end{array}$ & $\begin{array}{l}\text { GrupoST } \\
\text { Google } \\
\text { LatamAirl. } \\
\text { CocaCola } \\
\text { Facebook } \\
\text { SAP } \\
\text { GrupoINSUD }\end{array}$ & $\begin{array}{l}\text { GrupoST } \\
\text { Google } \\
\text { LatamAirl. } \\
\text { Unilever } \\
\text { Microsoft } \\
\text { Walmart } \\
\text { Facebook } \\
\text { SAP } \\
\text { VISA } \\
\text { GrupoINSUD }\end{array}$ \\
\hline : & $\begin{array}{l}\text { Sodexo } \\
\text { Kimb.Clark } \\
\text { Hogar de Cristo } \\
\text { Bethia } \\
\text { Sup.ChileAC }\end{array}$ & $\begin{array}{l}\text { P.U.Católica } \\
\text { CorreosChile }\end{array}$ & $\begin{array}{l}\text { Sup.ChileAC } \\
\text { Kimb.Clark }\end{array}$ & $\begin{array}{l}\text { Kimb.Clark } \\
\text { P\&G }\end{array}$ & $\begin{array}{l}\text { Asech } \\
\text { Sodexo } \\
\text { P\&G } \\
\text { Kimb.Clark } \\
\text { BancoEstado } \\
\text { Fund.Luksic }\end{array}$ & $\begin{array}{l}\text { Asech } \\
\text { Sodexo } \\
\text { BancoEstado } \\
\text { Fund.Luksic } \\
\text { Kimb.Clark } \\
\text { Acc.Empresas }\end{array}$ & $\begin{array}{l}\text { Asech } \\
\text { Sofofa } \\
\text { Oracle } \\
\text { Acc.Empresas } \\
\text { Andess AG } \\
\text { GrupoLUKSIC } \\
\text { AguasAndinas }\end{array}$ & $\begin{array}{l}\text { Asech } \\
\text { Acc.Empresas } \\
\text { AguasAndinas } \\
\text { Andess AG } \\
\text { Sofofa } \\
\text { Oracle } \\
\text { Unilever }\end{array}$ \\
\hline 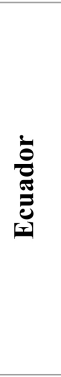 & $\begin{array}{l}\text { Nobis } \\
\text { Tonicorp } \\
\text { ElComercio } \\
\text { Microsoft } \\
\text { Corp.Favorita } \\
\text { Aerogal } \\
\text { FIEmpresarial } \\
\text { LasFragancias }\end{array}$ & $\begin{array}{l}\text { Nobis } \\
\text { Microsoft } \\
\text { ElComercio } \\
\text { Tonicorp } \\
\text { FIEmpresarial } \\
\text { LasFragancias } \\
\text { Movistar } \\
\text { AdrianaHoyos } \\
\text { Medeport } \\
\text { Renault }\end{array}$ & $\begin{array}{l}\text { Nobis } \\
\text { CocaCola } \\
\text { Tonicorp } \\
\text { ElComercio } \\
\text { AdrianaHoyos } \\
\text { DKManag. } \\
\text { Kimb.Clark } \\
\text { Pfizer } \\
\text { Tecnandina } \\
\text { LasFragancias } \\
\text { Microsoft } \\
\text { Corp.Favorita } \\
\text { Sweet\&Coffee }\end{array}$ & - & $\begin{array}{l}\text { Nobis } \\
\text { CocaCola } \\
\text { Corp.Favorita } \\
\text { DKManag. } \\
\text { Tonicorp } \\
\text { AdrianaHoyos } \\
\text { Pfizer } \\
\text { LasFragancias } \\
\text { Conecel } \\
\text { J\&JHCons. } \\
\text { ElComercio }\end{array}$ & $\begin{array}{l}\text { Nobis } \\
\text { DKManag. } \\
\text { Corp.Favorita } \\
\text { CocaCola } \\
\text { AdrianaHoyos } \\
\text { Tonicorp } \\
\text { Pfizer } \\
\text { Nestlé } \\
\text { CBCTesalia } \\
\text { ElComercio } \\
\text { C.I.Guayaquil } \\
\text { LasFragancias }\end{array}$ & $\begin{array}{l}\text { Nobis } \\
\text { DKManag. } \\
\text { Yanbal } \\
\text { CBCTresalia } \\
\text { AdrianaHoyos } \\
\text { Tipti } \\
\text { Pfizer } \\
\text { Impaqto } \\
\text { ElComercio } \\
\text { LasFragancias } \\
\text { C.I.Guayaquil } \\
\text { Nestlé } \\
\text { Kimb.Clark } \\
\text { CocaCola } \\
\text { Tonicorp } \\
\text { Frutadeli }\end{array}$ & - \\
\hline 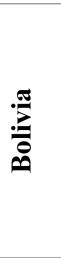 & $\begin{array}{l}\text { Yanbal } \\
\text { BancoFie } \\
\text { FarmaCorp } \\
\text { B.Canedo } \\
\text { Asfade } \\
\text { TropicalTours } \\
\text { GTB } \\
\text { Petrobras } \\
\text { LosTajibos }\end{array}$ & $\begin{array}{l}\text { Yanbal } \\
\text { FarmaCorp } \\
\text { Transierra } \\
\text { Asfade } \\
\text { Fidalga } \\
\text { BancoUnión } \\
\text { BancoSol } \\
\text { GTB } \\
\text { B.Canedo } \\
\text { BancoFie } \\
\text { P\&G }\end{array}$ & $\begin{array}{l}\text { Yanbal } \\
\text { FarmaCorp } \\
\text { BancoUnión } \\
\text { BPOCenter } \\
\text { IOL } \\
\text { B.Canedo } \\
\text { BancoFie } \\
\text { Incerpaz } \\
\text { Fidalga }\end{array}$ & $\begin{array}{l}\text { FarmaCorp } \\
\text { Yanbal } \\
\text { BancoUnión } \\
\text { IOL } \\
\text { Ferr.Oriental } \\
\text { LosTajibos } \\
\text { Asfade } \\
\text { Comteco }\end{array}$ & $\begin{array}{l}\text { FarmaCorp } \\
\text { BancoUnión } \\
\text { Yanbal } \\
\text { Kimb.Clark } \\
\text { Cerabol } \\
\text { Unifranz } \\
\text { Ferr.Oriental } \\
\text { IOL } \\
\text { Mitsuba } \\
\text { BPOCenter } \\
\text { L.Castellanos }\end{array}$ & $\begin{array}{l}\text { FarmaCorp } \\
\text { Yanbal } \\
\text { Kimb.Clark } \\
\text { Cerabol } \\
\text { L.Castellanos } \\
\text { BeautyPlaza } \\
\text { Coronilla } \\
\text { Unifranz } \\
\text { BPOCenter }\end{array}$ & $\begin{array}{l}\text { FarmaCorp } \\
\text { Yanbal } \\
\text { Kimb.Clark } \\
\text { Cerabol } \\
\text { Solquifar } \\
\text { BeautyPlaza } \\
\text { Unifranz } \\
\text { Fridolin } \\
\text { L.Castellanos } \\
\text { Coronilla }\end{array}$ & - \\
\hline
\end{tabular}




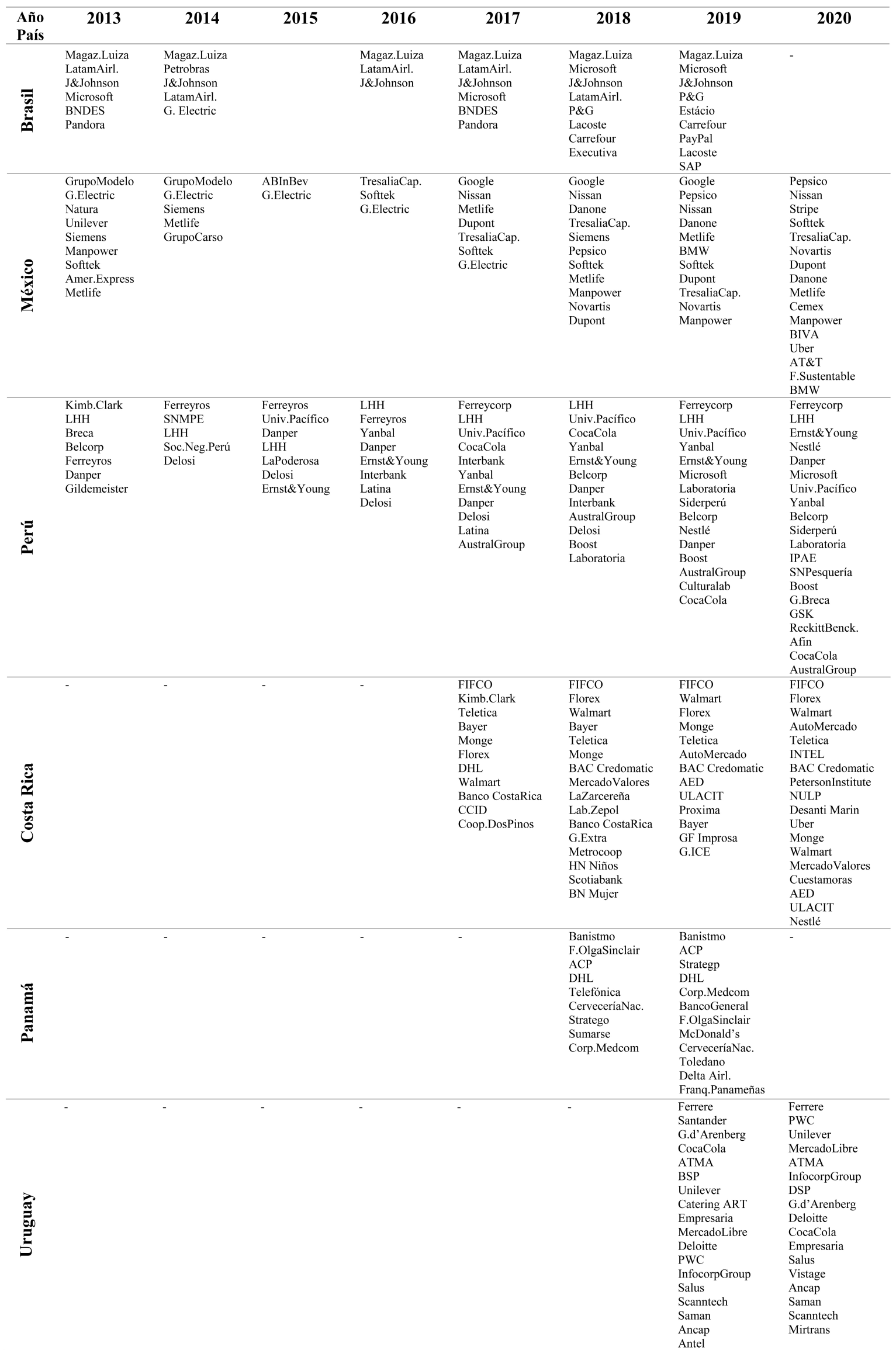


En relación a la composición de los rankings, puede observarse que en España las empresas reconocidas por el liderazgo de algunas de sus líderes son, en su mayoría, multinacionales extranjeras con intensa presencia internacional — por ejemplo, Google, Coca Cola, Microsoft, IBM, McDonald's, Facebook, Amazon, ING, HP, etc.seguidas de las grandes multinacionales y transnacionales españolas que, en general, lideran sus respectivos sectores en su país de origen - por ejemplo, Santander, Iberdrola, FCC o Inditex - y, por último, algunas pocas empresas españolas que, aunque tienen actividad internacional, operan generalmente en un ámbito nacional — por ejemplo, Codorniú, Holaluz, ESADE, Día, etc. En el caso de Argentina, los primeros listados sólo posicionan a las multinacionales Google, Microsoft y General Motors y más multinacionales extranjeras van sumándose a las listas de años siguientes, incluyendo éstas sólo dos compañías argentinas — Grupo ST y Grupo INSUD. Brasil también cuenta con un mayor número de empresas multinacionales extranjeras en sus listados únicamente Magazine Luiza, Petrobras, BNDES, Executiva y Estácio son compañías brasileñas. Por su parte, México tiene un listado bastante mixto, aunque las multinacionales extranjeras superan en número ligeramente a las empresas mexicanas.

Por el contrario, en Colombia, Chile, Ecuador, Bolivia, Perú, Costa Rica, Panamá y Uruguay predominan las empresas que operan principalmente en el ámbito nacional. Los listados colombianos están todos compuestos por empresas con origen en el país, a excepción de las multinacionales extranjeras
Google, Pepsico, P\&G y Discovery Networks. Chile también incluye una mayor parte de empresas chilenas, posicionando algún año únicamente a las multinacionales Kimberly Clark, P\&G, Oracle y Unilever. Lo mismo ocurre en los rankings ecuatorianos, que sólo dan cabida a algunas multinacionales -Microsoft, Coca Cola, Kimberly Clark, Pfizer y Nestléy a la transnacional peruana Yanbal. También Bolivia cuenta en los listados con empresas bolivianas en su mayor parte, apareciendo en algunos años las multinacionales $P \& G$ y Kimberly Clark y las transnacionales Yanbal y Petrobras. De igual forma, la clasificación peruana está compuesta por muchas empresas de origen nacional, con algunas pocas multinacionales y/o transnacionales - Microsoft, Coca Cola, Ernst\&Young, Lee Hecht Harrison, Yanbal, Nestlé, GSK y Reckitt Benckiser. Costa Rica cuenta con muchas más empresas nacionales, aunque incluye varias multinacionales como Kimberly Clark, Bayer, DHL, Walmart, Scotiabank, Intel, Uber y Nestlé. Panamá, a excepción de DHL, Telefónica, McDonald's y Delta Airlines, compone sus listados mayoritariamente con empresas panameñas. Por último, Uruguay también suma más empresas uruguayas y da cabida sólo a las multinacionales Coca Cola, Unilever, Deloitte, PWC y Santander.

Por su parte, la Tabla 3 resume, para cada compañía que conforma la muestra objeto de estudio, las distintas iniciativas y programas específicos que, durante el periodo 2013-2020, se dirigen a impulsar el liderazgo femenino y fomentar su representación en las distintas posiciones directivas.
Tabla 3 Iniciativas $y$ programas a favor del liderazgo femenino. Fuente: Elaboración propia

\begin{tabular}{|c|c|c|}
\hline Compañía & Posiciones de liderazgo & Consejos de Administración \\
\hline Microsoft & $\mathrm{X}$ & $\mathrm{X}$ \\
\hline Siemens & Cuotas & Cuotas \\
\hline McDonald's & Cuotas & $\mathrm{X}$ \\
\hline Santander & $\begin{array}{l}\text { Sumando Talento, Take The } \\
\text { Lead, Santander Me y Cuotas }\end{array}$ & Cuotas \\
\hline Manpower & $\begin{array}{l}\text { Women's Leadership } \\
\text { Initiative y Cuotas }\end{array}$ & Cuotas \\
\hline Telefónica & $\begin{array}{c}\text { Women in Leadership y } \\
\text { Cuotas }\end{array}$ & $\mathrm{X}$ \\
\hline Coca Cola & $\begin{array}{c}\text { Global Women's Initiative } \\
\text { y Cuotas }\end{array}$ & Cuotas \\
\hline Facebook & Women LEAD y Women LEAP & $\mathrm{X}$ \\
\hline Google & Polaris & $\mathrm{X}$ \\
\hline $\begin{array}{l}\text { General } \\
\text { Electric }\end{array}$ & $\mathrm{X}$ & $\mathrm{X}$ \\
\hline P\&G & Cuotas & Cuotas \\
\hline Pepsico & Cuotas & $\mathrm{X}$ \\
\hline $\begin{array}{l}\text { Latam } \\
\text { Airlines }\end{array}$ & $\mathrm{X}$ & $\mathrm{X}$ \\
\hline $\begin{array}{l}\text { Kimberly } \\
\text { Clark }\end{array}$ & $\begin{array}{l}\text { Strengthening the Business } \\
\text { With Women Leaders }\end{array}$ & $\mathrm{X}$ \\
\hline Unilever & $\begin{array}{c}\text { Women's Leadership } \\
\text { Develpment Programme y } \\
\text { Cuotas }\end{array}$ & $\mathrm{X}$ \\
\hline Nestlé & $\begin{array}{c}\text { Gender Balance Acceleration } \\
\text { Plan y Cuotas }\end{array}$ & $X$ \\
\hline Yanbal & $\mathrm{X}$ & $\mathrm{X}$ \\
\hline Petrobras & $\mathrm{X}$ & $\mathrm{X}$ \\
\hline Walmart & $\begin{array}{l}\text { Leadership Development } \\
\text { Women's Program }\end{array}$ & $\mathrm{X}$ \\
\hline DHL & $\begin{array}{c}\text { Women in Management y } \\
\text { Cuotas }\end{array}$ & $X$ \\
\hline
\end{tabular}


Microsoft reconoce en todos sus Global Diversity \& Inclusion Reports, con especial atención desde 2016, su compromiso para fomentar la diversidad de género en el ámbito tecnológico con programas como DigiGirlz o Codess, siendo su iniciativa Women@Microsoft, dirigida a proporcionar a las mujeres más recursos para su desarrollo profesional, la más destacada. Este compromiso ha dado lugar a un ligero incremento de la representación femenina en los puestos tecnológicos — del $17,1 \%$ de mujeres en 2016 al $22,8 \%$ en 2020 - y en su plantilla total - del $25,5 \%$ en 2016 al $28,6 \%$ en 2020 . También ha mejorado la presencia femenina en los puestos directivos y de gestión. Concretamente, en 2016 había un $21,7 \%$ de mujeres managers y un 18\% ocupando puestos directivos y esos porcentajes ascienden al $26,3 \%$ y $21,1 \%$, respectivamente, en 2020. El Consejo de Administración también ha tendido a una composición más diversa y, en 2020, de los 12 miembros que lo componen, 5 son mujeres, lo que supone una representación femenina del 41,6\%. No obstante, a pesar del avance, la compañía no cuenta con ningún programa específico para impulsar el liderazgo femenino ni fija cuotas de género para alcanzar una mínima representación en sus distintas posiciones jerárquicas.

Siemens AG firma en 2016 los Principios para el Empoderamiento de las Mujeres - primera iniciativa mundial de la ONU enfocada a promover el talento femenino en las empresas. También en ese año comienza a publicar medidas de diversidad en sus Diversity Fact Sheets y, de acuerdo con la German Law for Equal Participation of Women an Men in Management Positions in the Private and Public Sectors - establece que el 30\% de los puestos estén ocupados por mujeres-, fija para 2017 el objetivo de alcanzar la proporción de $2 / 7$ de mujeres en el Consejo de Administración y un porcentaje mínimo del $10 \%$ en cada uno de los dos niveles de gestión por debajo del Comité de Dirección (esto es, para las senior managers y managers). A cierre de 2017, las mujeres representan el 23\% de la plantilla total, incluyendo el Consejo de Administración a 2 mujeres (de un total de 8) y existiendo un $15 \%$ de managers y un $11 \%$ de senior managers. Tras cumplir parcialmente los objetivos marcados el año anterior, y sin mostrar cambios en el Consejo de Administración, Siemens fija el objetivo de lograr en 2022 un porcentaje mínimo de mujeres del $20 \%$ en los dos niveles de gestión inferiores al Comité de Dirección. En 2018 mantiene la misma composición para el Consejo de Administración - proporción 2/8, que equivale al $25 \%$ - pero eleva al $30 \%$ la presencia de mujeres en el Comité de Dirección, cumpliendo con la cuota establecida por el gobierno alemán. En 2019 incrementa ligeramente las cifras de managers y senior managers - $16 \%$ y $12 \%$, respectivamente - y mantiene la representación femenina en el Comité de Dirección. En 2020 existe un ligero avance en las posiciones directivas: el $24 \%$ de la plantilla son féminas, ocupando éstas el 17\% y 14\% de los puestos de managers y senior managers, respectivamente; sin embargo, se reduce la proporción de mujeres en el Consejo de Administración — de un total de 7 miembros, solo uno es mujer-y es por ello que vuelve a fijar el objetivo de que, en 2022, al menos el $25 \%$ de los consejeros sean mujeres.

McDonald's Corporation muestra información detallada sobre diversidad e inclusión a partir de 2018, cuando su plantilla está compuesta en un $54 \%$ por mujeres y éstas ocupan el 38\% de los puestos directivos. En 2019 firma los Principios para el Empoderamiento de las Mujeres e implanta, dentro de su programa Archways to Opportunity, la iniciativa Women in Tech con la intención de apoyar una representación más amplia de mujeres en los puestos relacionados con la tecnología. Aunque a lo largo de ese año el porcentaje de mujeres en plantilla no varía, se incrementa hasta el 39\% la representación femenina en puestos directivos. Con el $41 \%$ de los puestos directivos ocupados por mujeres en 2020, la compañía muestra su compromiso por mejorar la diversidad en todos los niveles en 2023 y fija el objetivo de que ellas representen el $45 \%$ de las posiciones directivas en 2025, habiéndose propuesto lograr a nivel mundial la paridad de género en puestos de liderazgo para finales de 2030. En cuanto al Consejo de Administración, a pesar de que sólo 3 de los 13 miembros son mujeres, no fija ningún objetivo al respecto.

En sus publicaciones de 2013, Banco Santander subraya la importancia de la diversidad de género y de fomentar la igualdad de oportunidades entre hombres y mujeres, contando a cierre de ese año con un $54,7 \%$ de mujeres en plantilla y con un Consejo de Administración compuesto en un $18,8 \%$ por consejeras -3 mujeres de un total de 16 miembros. En 2014 pone en marcha los programas Sumando Talento y Take The Lead - dirigidos a mujeres en posiciones directivas o predirectivas de todos los países para impulsar el desarrollo del talento y liderazgo femenino-y fija en el 25\% el porcentaje mínimo de mujeres en el Consejo de Administración. Ambas iniciativas, manteniéndose un 55\% de mujeres en plantilla, contribuyen a incrementar en 2015 la representación femenina en las posiciones de liderazgo: el $17,4 \%$ son directivas y el $33,3 \%$ de los miembros del Consejo de Administración son mujeres. A principios de 2016, de acuerdo con el Código de Buen Gobierno de las Sociedades Cotizadas en España, eleva el objetivo de mujeres en el Consejo hasta el $30 \% \mathrm{y}$, adicionalmente, implanta el programa Santander Me con el objetivo de acompañar el empoderamiento de las mujeres. Al cierre de ese año, con un $40 \%$ de representación femenina en el Consejo - 6 de un total de 15-, supera el objetivo marcado y consigue un ligero incremento de mujeres en posiciones directivas — hasta el 19,4\%. En los dos años siguientes se reduce mínimamente el tamaño del Consejo y la proporción de consejeras - 35,7\% y $33 \%$ en 2017 y 2018, respectivamente- aumentando, no obstante, el porcentaje de mujeres en puestos directivos hasta el $20,5 \%$ en 2018 y fijando el nuevo objetivo de lograr el $30 \%$ de mujeres directivas en 2025. En 2019 firma los Principios para el Empoderamiento de las Mujeres y en 2020 se adhiere al programa Target Gender Equality lanzado por el Pacto 
Mundial de Naciones Unidas para aumentar la representación de las mujeres en los Consejos de Administración y los puestos de dirección ejecutiva. Contando en 2020 con un $40 \%$ y $23,7 \%$ de mujeres en el Consejo de Administración y puestos directivos, respectivamente, se compromete a alcanzar en 2021 una composición del Consejo que incluya entre un $40 \%$ y $60 \%$ de consejeras.

Manpower Group presenta, desde el principio del periodo, altas cifras de representación femenina en todos sus niveles organizativos. En 2013, alrededor del 65\% de los empleados son mujeres y, en cuanto a la presencia femenina en posiciones de liderazgo, el porcentaje de féminas para el total de managers y senior managers es del 55\% y 34\%, respectivamente, teniendo el Consejo de Administración una proporción de mujeres 3/10. Mostrando en los años siguientes porcentajes de representación femenina ligeramente superiores en todos los niveles, desde 2015 es incluida en el Pax Ellevate Global Women's Leadership Fund como una de las compañías con mayor retorno de la inversión asociada a la diversidad de género y al liderazgo de las mujeres. En 2017, el 69\% de empleados son mujeres, existiendo un $57 \%$ de managers, un $33 \%$ de senior managers y un $29 \%$ de ejecutivas y contando el Consejo de Administración con un $42 \%$ de consejeras. El lanzamiento de Women's Leadership Initiative en 2018 y el incremento de la representación femenina en el Consejo de Administración hasta el $45 \%$ favorece que la compañía esté en 2019 incluida en los listados Women's Choice Awards' Best Companies to Work for Women y Forbes Best Employers for Women. Aunque a cierre de 2020 su Consejo de Administración ha reducido la representación femenina hasta el 38,5\%, ha recibido el Women on Boards Honor Roll y se ha comprometido a incrementar hasta el $40 \%$ la representación femenina en todas las posiciones de liderazgo en 2024.

Telefónica cuenta en 2013 con un 38,3\% de mujeres en plantilla, ocupando éstas el $21 \%$ de puestos directivos y el $5,56 \%$ de asientos en el Consejo de Administración - 1 consejera y 17 consejeros. Con el objetivo de incrementar la visibilidad de las mujeres en puestos de liderazgo, implanta a nivel global en 2012 el programa Women in Leadership (aún vigente en 2020), el cual es complementado en años siguientes con distintas acciones a nivel local - como, por ejemplo, Talentia en España, Mujeres\&Líderes en Colombia, Talento Femenino en México o Women Network en Perú. En 2015 y 2016 el Consejo de Administración incrementa la representación femenina hasta el 11,1\% y 17,6\%, respectivamente, y dado que los porcentajes de mujeres en plantilla y puestos directivos muestran mínimas reducciones, fija el objetivo de incrementar el porcentaje de mujeres directivas hasta el $30 \%$ en 2020 . Ante tal compromiso, el porcentaje de directivas pasa del $21,5 \%$ en 2017 al $23,3 \%$ y $26 \%$ en 2018 y 2019, respectivamente. La compañía cierra 2020 con un $38 \%$ de mujeres en plantilla y un $29,4 \%$ de mujeres en el Consejo de Administración - 5 de un total de 17 -, siendo el porcentaje de mujeres directivas del $27 \%$.
Desde que en 2007 Coca Cola Company crea el Global Women's Leadership Council e implanta Global Women's Initiative para promover el talento femenino y garantizar el equilibrio de género en toda la organización, las cifras de representación femenina en plantilla se han mantenido alrededor del $47 \%$ a lo largo del periodo y las referidas a las posiciones de liderazgo han ido en aumento. La participación desde 2011 en la campaña 2020 Women on Boards — para lograr que las mujeres ocupen al menos el $20 \%$ de los asientos del Consejo de Administración en 2020-y la creación de Women in Leadership Program en 2013 ha contribuido a la promoción de los líderes femeninos desde entonces: en 2016 un $43 \%$ y $31,7 \%$ de managers y senior managers eran mujeres, respectivamente, y en 2018 esos porcentajes ascienden al 45,1\% y 32,1\%. Su Consejo de Administración, integrado desde 2013 por 4 mujeres y 11 hombres - $26,6 \%$ de representación femenina-, suma en 2018 un nuevo miembro mujer, lo que supone un incremento hasta el 31,25\%. En 2019 ha mostrado su compromiso para alcanzar próximamente el objetivo de diversidad 50/50 en las posiciones de liderazgo, cerrando el año con un 48,4\% y 33,5\% de managers y senior managers mujeres, respectivamente, y una representación femenina en el Consejo de Administración del 30,7\% - 4 mujeres de un total de 13 . Finalmente, cierra 2020 con un $38 \%$ de mujeres en plantilla, alrededor del $50 \%$ de mujeres managers y un Consejo de Administración compuesto por 5 mujeres de un total de 13 , lo que supone un $38,5 \%$.

Facebookreconocequelasmujeres estáninfrarrepresentadas en todos los niveles organizativos de la compañía y por ello ha puesto en marcha los programas Women LEAD y Women LEAP — el primero está diseñado para brindar apoyo a las mujeres líderes y el segundo extiende el impacto de Women LEAD a la próxima generación de mujeres que pueden convertirse en líderes dentro de la compañía. Desde que en 2014 las mujeres representaran el $31 \%$ de la plantilla total y el 16,6\% de los miembros del Consejo de Administración -2 de un total de 12-, los porcentajes de representación femenina han crecido: en 2020 el $37 \%$ de empleados y el $40 \%$ de miembros del Consejo de Administración — 4 de un total de 10 - son mujeres.

Google, con una plantilla muy masculinizada, mantiene a lo largo del periodo cifras similares en lo que a representación femenina se refiere - en 2014 el 30,6\% de los empleados son mujeres y en 2020 ellas representan el 32\%. Un mayor avance se consigue en lo que a posiciones de liderazgo se refiere - del 20,8\% en 2014 al 25,5\% en 2018 y al 26,7 en 2020 - lo que ha podido deberse al lanzamiento del programa Polaris para apoyar el desarrollo profesional de mujeres líderes en ascenso. La presencia femenina en el Consejo de Administración también ha mejorado ligeramente: en 2013 el $18,8 \%$ de los asientos estaban ocupados por mujeres y en 2020 este porcentaje asciende al $23 \%$. 
General Electric presenta también una fuerza laboral mayoritariamente masculina. Con tan sólo con un 18\% de mujeres en plantilla en 2016, en 2017 anuncia su compromiso a largo plazo de lograr una representación de género 50/50 en plantilla. No obstante, el avance en cuatro años es mínimo: en 2020 las mujeres representan el $21,9 \%$ de la plantilla. Por lo que respecta al liderazgo femenino, participa en la iniciativa mundial Women's Network y en 2015 es reconocida con el distintivo Best Places for Women \& Diverse Managers to Work, alcanzando en 2020 un $26 \%$ de representación femenina en las posiciones de liderazgo. Su Consejo de Administración, que en 2013 cuenta con un 28\% de mujeres, reduce la presencia femenina en 2017 hasta el 17\%, pero vuelve a incrementarla hasta el $36,36 \%$ en 2020 .

Desde hace años, $P \& G$ ha puesto en marcha campañas como \#WeSeeEqual o Women at Work: Myth vs. Reality para promover la inclusión y diversidad de género en los lugares de trabajo y cuenta con un Corporate Women's Leadership Team (CWLT) que se enfoca al avance del liderazgo femenino. En 2015 posee alrededor de un 36\% de mujeres empleadas y un $45 \%$ de managers femeninas, contando con una representación del $36,36 \%$ en el Consejo de Administración. Desde entonces, muestra como prioridad alcanzar la proporción 50/50 en todos los niveles jerárquicos, lo que le ha llevado en 2020 a tener una fuerza laboral compuesta por un $40 \%$ de mujeres, un $45 \%$ de managers femeninos y un Consejo de Administración con un $42 \%$ de representación femenina. Su compromiso con la diversidad ha posicionado a la compañía en los últimos años en los listados Best Company for Female Executives NAFE y Top Companies for Executive Women y ha contribuido a incluir a varias de sus líderes en los rankings Fortune Most Powerful Women (International) y Women Inc. Most Influential Corporate Directors.

Pepsicofirmaen 2013 los Principios paraelEmpoderamiento de las Mujeres, año en el que cuenta con un 33\% de managers femeninos y un Consejo de Administración compuesto por el 30,7\% de mujeres. En 2015 lanza el programa PepsiCo's Transformational Leadership Program con la intención, entre otras, de brindar a las mujeres habilidades de liderazgo, lo que favorece, en adelante, el incremento de managers femeninos - concretamente, desde el 38\% en 2016 hasta el $41,2 \%$ en 2020. El Consejo de Administración cuenta en 2020 con el $23,1 \%$ de miembros femeninos pero la compañía ha subrayado el objetivo de alcanzar el $50 \%$ de representación femenina en todas sus posiciones de liderazgo para 2025.

Latam Airlines Group expone en sus publicaciones brevemente su compromiso con la diversidad e igualdad pero no especifica ninguna política ni acción al respecto. Desde 2015 la composición por género de su plantilla se mantiene estable - 38,6\% de empleadas en 2015 y $39,5 \%$ en 2019 y el porcentaje de managers femeninas ligeramente se incrementa, pasando del $27,22 \%$ al 29,9\%, respectivamente. En cuanto al Consejo de Administración, integrado en 2015 por 9 miembros, todos ellos masculinos, éste modifica su composición a partir de 2018 , contando con 1 consejera y 8 consejeros, lo que supone una representación femenina del $11,1 \%$.

Desde que Kimberly Clark lanzara en 2009 la iniciativa Unleash Your Power: Strengthening the Business With Women Leaders para impulsar el liderazgo femenino y creara poco antes de 2013 el puesto de Global Diversity Officer con la intención de diseñar e impulsar estrategias a favor de la diversidad e inclusión, la compañía ha incrementado la representación femenina en los puestos de gestión y liderazgo. Aunque el porcentaje de empleadas desciende a lo largo del periodo - del 35,6\% en 2013 al 30\% en 2018-, las managers aumentan ligeramente, pasando del $30,3 \%$ en 2013 al 33,4 en 2018. Este incremento lleva a la compañía a situarse en 2018 y 2019 en la clasificación NAFE Top 70 Companies for Executive Women. Por lo que respecta al Consejo de Administración, integrado en 2013 por un 25\% de consejeras, modifica ligeramente su composición en años siguientes pero termina 2020 en esa misma proporción.

Con el objetivo de construir una compañía equilibrada en relación con el género y con especial enfoque en las posiciones de liderazgo, Unilever pone en 2012 en marcha Women's Leadership Develpment Programme en colaboración con INSEAD Unilever Four Acres Consortium. Desde entonces, en su Unilever Sustanaible Living Plan, dentro de su estrategia de diversidad e inclusión, establece la ambición por alcanzar en 2020 el 50\% de mujeres managers, lo que favorece el incremento de las cifras en años siguientes - desde el 45\% en 2015 al 50,7\% en 2019. A lo largo del periodo, la composición de la plantilla ha variado mínimamente - representación femenina en torno al 35\%y el Consejo de Administración, integrado en 2016 por 6 mujeres y 8 hombres (43\%), pasa desde entonces a contar con 5 mujeres de un total de 13 (38\%).

Desde que en 2008 estableciera como prioridad incrementar la presencia femenina en todos los niveles (especialmente en los puestos de managers y senior leaders) y firmara en 2013 los Principios para el Empoderamiento de las Mujeres, Nestlé, que cuenta con una plantilla a lo largo del periodo compuesta aproximadamente por el $34 \%$ de féminas, pasa de tener alrededor de un 30\% de managers femeninos en 2013 a un 37,5\% en 2017. En 2018 el aumento es más significativo, ocupando posiciones de liderazgo un $43,2 \%$ de mujeres y representando éstas el $31,8 \%$ de los senior managers. En 2019, con el lanzamiento del Gender Balance Acceleration Plan para conseguir un mayor equilibrio de género en la plantilla global e incrementar la presencia femenina en los más altos cargos, anuncia el objetivo de lograr en 2022 que el $30 \%$ de los puestos senior ejecutivos estén ocupados por mujeres. Su Consejo de Administración, integrado durante todo el periodo por 14 miembros, muestra pocas variaciones, contando con 4 consejeras en 2013 y 5 en 2020 . 
Yanbal International, aunque publica su Política Global de Diversidad e Inclusión, no ofrece apenas información relacionada con la composición de su plantilla . A cierre de 2019 cuenta con un 55\% de mujeres en plantilla y el 57\% de las posiciones de liderazgo están ocupados por féminas. $\mathrm{Su}$ Consejo de Administración está compuesto por 1 mujer y 2 hombres.

Petrobras firma en 2010 los Principios para el Empoderamiento de las Mujeres y en 2013 su plantilla la componen un $17,1 \%$ de empleadas y un $15,4 \%$ de managers femeninas, con $2 / 10$ de representación femenina en el Consejo de Administración. A lo largo del periodo, el porcentaje de mujeres en el total de la fuerza laboral se reduce ligeramente hasta el $16 \% \mathrm{y}$, del total de managers, las féminas llegan a representar un $18 \%$ en 2018 y 3/10 del Consejo de Administración. En 2019, manteniéndose el Consejo sin variaciones, cae ligeramente la presencia femenina en los puestos de managers, representando éstas el $16 \%$.

Walmart implanta en 2013 Leadership Development Women's Program (BOLD), dirigido a desarrollar el potencial de las directivas. Desde ese año, con una plantilla compuesta por un $57,2 \%$ de mujeres, incrementa ligeramente sus cifras de representación femenina en posiciones de liderazgo pasa del $40 \%$ de managers y $29,5 \%$ de senior managers femeninas en 2013 al $45 \%$ y $27,5 \%$, respectivamente, en 2020. La proporción de mujeres en plantilla y en el Consejo de Administración oscilan poco a lo largo del periodo, ya que la compañía cierra 2020 con aproximadamente el 55\% de mujeres en plantilla y su Consejo, con una representación femenina en 2013 del 23,5\%, está compuesto por un $27 \%$ de miembros femeninos -3 de un total de 11 .

DHL inicia en 2011 el proyecto Women in Management, el cual complementa a partir de 2014 con el programa Top Women con la finalidad de preparar a las mujeres para asumir puestos de liderazgo y desarrollar su potencial como líderes. En 2013 posee una plantilla con el $35,8 \%$ de empleadas y el $19,6 \%$ de managers femeninos. Desde entonces, la plantilla se ha mantenido con pocas variaciones $-34,4 \%$ de empleadas en 2019-, al igual que su Consejo de Administración proporción $1 / 7$ hasta 2018 y $1 / 8$ a partir de 2019-, mientras que el porcentaje de mujeres en posiciones de liderazgo ha ido ligeramente en aumento hasta situarse en 2019 en $22,2 \%$, año en el que ha fijado el objetivo de incrementar esa cifra al $30 \%$ en 2025 .

El análisis previo permite, a partir de los datos disponibles, confirmar que:

1) Las compañías que, en general, ofrecen un mayor volumen de información acerca de las políticas de diversidad e impulso del liderazgo femenino son las europeas, siendo las latinoamericanas en este sentido las más rezagadas.
2) Respecto a las políticas y estrategias vinculadas a diversidad, casi todas las compañías reportan un incremento de la inversión a tal respecto. Se observa que la gran mayoría de empresas cuenta con iniciativas o participa en campañas dirigidas a otorgar una mayor visibilidad, empoderar y activar profesionalmente a las mujeres en su conjunto pero, sin embargo, desciende el número de empresas que implantan programas que, de manera específica, se dirigen a impulsar sus trayectorias y carreras como líderes o que han establecido cuotas para incrementar la representación femenina en posiciones de liderazgo y Consejos de Administración.

3) Al final del periodo solo dos compañías están presididas por una mujer CEO — Santander y Yanbaly las mejores cifras de diversidad (en términos absolutos) se encuentran, para las posiciones de liderazgo, en Manpower, Coca Cola, P\&G, Unilever, Yanbal y Walmart y, para los Consejos de Administración, en Microsoft, Santander, P\&G y Facebook. Por el contrario, Siemens, Petrobras y DLH poseen las peores cifras de representación femenina en las posiciones de liderazgo, uniéndose a éstas también Latam Airlines en lo que al Consejo de Administración respecta.

4) Las compañías que presentan un mayor avance en posiciones de liderazgo son Telefónica, Pepsico, Nestlé y Google, con incrementos alrededor del 25\%. Los Consejos de Administración que más crecen en representación femenina son los de Santander, Telefónica y Facebook.

5) Las empresas que habiendo establecido cuotas a lo largo del periodo han cumplido sus objetivos son Santander y Unilever, y también Coca Cola y Siemens parcialmente. Telefónica, habiendo fijado el objetivo de alcanzar el 30\% de mujeres directivas en 2020, cierra ese año con una cifra del $27 \%$.

\section{Discusión}

En primer lugar, los resultados de explorar el ranking MERCO Líderes ha evidenciado la infrarrepresentación de las mujeres como líderes en todos los países iberoamericanos examinados. En concreto, España, Costa Rica, Perú y Uruguay son los que cuentan, al final del periodo, con una mayor presencia femenina. Las peores cifras pertenecen a Argentina, Chile, Bolivia y Brasil, siendo estos tres últimos los que menor avance consiguen desde 2013. Estos hallazgos, por un lado, están muy en línea con los que ha arrojado el ranking Global Gender Gap Index en los últimos años, estando España y Costa Rica en primeras posiciones - específicamente, en el puesto 8 y 13, respectivamente, del ranking de 2020 - y países como Bolivia, Chile y Brasil en posiciones más atrasadas — puesto 42, 57 y 92, respectivamente. Por otro lado, los resultados también 
guardan coherencia con los publicados por Deloitte (2019) en su estudio acerca de la representación femenina en diferentes posiciones organizativas y distintos países. España es, de los países examinados, el que mejores cifras presenta: las mujeres representan el 19,2\% de los Consejos de Administración, el 5,3\% como presidentas del Consejo y el 1,3\% como CEOs. Sin embargo, los porcentajes para Argentina son, respectivamente, de 4,7\%, 0\% y 0\%; para Brasil son de $8,6 \%, 6,55 \%$ y $0,8 \%$ y para Chile son de $7,7 \%$, $0 \%$ y $0 \%$.

Esto sugiere que existen ciertas diferencias entre España y el resto de países iberoamericanos que pueden atribuirse tanto a factores legislativos como culturales. En cuanto a los factores legislativos, si bien todos los países latinoamericanos en las últimas décadas han ido introduciendo cuotas y adoptando objetivos para impulsar el avance de las mujeres en los órganos nacionales e instituciones gubernamentales y en los procedimientos electorales, al igual que España, pocos lo han hecho en la esfera empresarial. A este respecto, España cuenta, desde 2015, con el Código de Buen Gobierno de las Sociedades Cotizadas - la CNMV fijó el objetivo de que en 2020 el número de consejeras representara, al menos, el 30\% del total de los miembros del Consejo de Administracióny en 2019 ha promulgado el Real Decreto-Ley 6/2019 de Medidas urgentes para la garantía de igualdad de trato y de oportunidades entre mujeres y hombres en el empleo y la ocupación, el cual obliga a las PYMES de entre 50 y 250 trabajadores a negociar y contar con un Plan de Igualdad, hasta entonces sólo obligatorio para empresas con más de 250 trabajadores en plantilla. Además, la modificación del Código de Buen Gobierno en 2020 ha elevado hasta el 40\% el porcentaje de mujeres consejeras en 2022. Por el contrario, los países latinoamericanos no cuentan con medidas para favorecer la representación femenina en las posiciones de liderazgo de las compañías —únicamente Colombia ha fijado una cuota del $30 \%$ para empresas estatales que no aplica a empresas privadas (Deloitte, 2019; Campos-García, Olivas-Luján y Zúñiga-Vicente, 2019). Por lo que respecta a los factores culturales, la Comisión Económica para América Latina y el Caribe asegura en estos países la persistencia de más barreras estructurales y patrones culturales patriarcales y discriminatorios - con frecuencia, dichas barreras suponen una resistencia a la reglamentación y aplicación de las leyes vigentes (CEPAL, 2020). De ahí que todos los países latinoamericanos estén por muy detrás de España en la lista de países ordenados según el Índice de Desarrollo Humano que elabora el Programa de las Naciones Unidas para el Desarrollo (PNUD) con la intención de medir la desigualdad de género.

En segundo lugar, respecto a las mujeres líderes (y sus respectivas compañías) que aparecen en los rankings MERCO a lo largo del periodo, llaman la atención dos cuestiones. La primera de ellas es la escasa variación que sufre la composición del listado con el paso de los años en algunos países; es decir, año tras año son nombradas, con pocas alteraciones, las mismas líderes que, eso sí, se mueven por las distintas posiciones del ranking - no se percibe relación alguna entre las posiciones ocupadas más avanzadas y mejores cifras de diversidad en sus compañías. Esto sucede, de manera más acusada, en Argentina y Brasil, seguidos de Ecuador, Colombia y España. La segunda se refiere a la tipología de empresas que componen el ranking de cada país: mientras que en España, Argentina, Brasil y México gran parte de las compañías que forman el listado son multinacionales, el resto de países incluye más empresas de ámbito nacional o transnacionales con origen nacional. Esta composición puede estar en parte influida por el grado de apertura comercial de cada economía.

En tercer lugar, en relación con el análisis de las memorias corporativas de las compañías que, habiendo sido incluidas en el ranking MERCO Líderes, muestran información detallada sobres sus políticas e iniciativas de diversidad, existen ciertos aspectos que pueden considerarse objeto de discusión. Primeramente, aunque no existe un patrón totalmente claro, sí pueden identificarse algunas relaciones. Por un lado, las compañías que muestran mejores cifras de diversidad en posiciones de liderazgo y Consejos de Administración, conjuntamente, son las que más políticas, programas y objetivos han adoptado. Éstas son P\&G, Coca Cola, Manpower y Santander, todas ellas compañías que cuentan con plantillas, en general, más feminizadas. También Pepsico, Kimberly Clark, Unilever, Nestlé o Walmart, compañías que operan en sectores con una fuerza laboral más feminizada, cuentan con programas específicos de fomento del liderazgo femenino - la mayoría de ellas han sido reconocidas por sus iniciativas para acelerar el progreso de las mujeres en los lugares de trabajo por Catalist Awards y han sido incluidas en el Bloomberg Gender Equality Index en uno o varios años. No obstante, compañías pertenecientes a sectores más masculinizados como el energético y el tecnológico - principalmente, Telefónica, Facebook, Google y Siemens - han tomado la iniciativa para implantar numerosas acciones en pro de la diversidad en los puestos directivos.

Por otro lado, las multinacionales latinoamericanas examinadas manifiestan prioridades y objetivos menos ambiciosos en materia de diversidad, siendo una clara evidencia que ninguna de ellas cuenta con ningún programa orientado al impulso del liderazgo de las mujeres. Este hallazgo está en línea, como se ha comentado anteriormente, con la ausencia de legislación (o una legislación menos estricta) y con una cultura nacional más rezagada en materia de género que permea al plano empresarial.

Finalmente, en relación a las cuotas, queda comprobar si las compañías que han fijado cuotas para alcanzar en años posteriores a 2020 por ejemplo -McDonald's, Santander, Manpower, General Electric, P\&G, Nestlé o Siemenscumplen sus objetivos. En cualquier caso, a partir de la información disponible, parece evidente el efecto positivo de las cuotas sobre el progreso de las mujeres en el acceso y la ocupación de posiciones de liderazgo. Sin embargo, debe 
tenerse en cuenta que las cuotas de género, dado que pueden existir otras variables explicativas, pueden no funcionar de la misma manera en todas las compañías. No obstante, las cuotas siguen generando un amplio debate a partir de los argumentos que sostienen que pueden dar lugar a una discriminación inversa o que restan mérito y menosprecian la contribución y valía de las mujeres que realmente merecen ocupar puestos de responsabilidad y liderazgo.

\section{Conclusiones}

Pese al incremento de la representación femenina en el plano laboral durante las últimas décadas, las mujeres siguen enfrentando numerosas barreras para escalar y avanzar posiciones en los puestos más altos de las organizaciones. La persistencia de ciertos estereotipos y roles de género sigue suponiendo un obstáculo para que las mujeres lideren y ocupen puestos de máxima responsabilidad. McKinsey (2018) revela que, en la mayoría de los casos, las mujeres son promocionadas en función del rendimiento, mientras que la promoción masculina se hace en base al potencial. Esto sugiere que las mujeres inician el ascenso una vez demostrada de sobra su valía, debiendo hacer más esfuerzos para conseguir, al menos, las mismas oportunidades laborales que los hombres.

Ellas son minoría en la mayoría de posiciones de liderazgo de todas las compañías del mundo y, además de ser pocas, su visibilidad también es escasa. Así se constata tras el examen de los datos arrojados por el ranking MERCO Líderes en países iberoamericanos. A pesar del incremento de las cifras durante 2013-2020, la baja visibilidad y representación femenina es indicativa de las disparidades de género que existen en el panorama empresarial. Es por ello que, en los últimos años, muchas compañías, con la intención de contribuir a los ODS de la Agenda 2030, han reconocido el incremento de la inversión en las políticas y estrategias de diversidad, poniendo en marcha distintas iniciativas a fin de promover la inclusión y fomentar el liderazgo femenino.

Los resultados aquí obtenidos dan lugar a ciertas consideraciones que implican a los tres agentes que pueden favorecer el progreso de las mujeres en la esfera empresarial - autoridades gubernamentales, empresas y sociedad. Por lo que respecta a las autoridades gubernamentales, el diseño de medidas que obliguen o dirijan a las empresas con origen en sus respectivos países hacia una mayor diversidad en los puestos directivos y hacia una mayor homogeneidad y transparencia en la publicación de información relacionada con el género podría favorecer un compromiso más firme y real con el avance deseado. En este sentido, cabe destacar que en España la Ley de Información No Financiera y Diversidad 11/2018 insta a las compañías a incluir en sus publicaciones asuntos relativos a la política de diversidad y a las medidas adoptadas para promover la igualdad de trato y oportunidades entre hombres y mujeres. También el Consejo de Negocios Internacionales del Foro Económico Mundial ha lanzado en 2020 'Measuring Stakeholder Capitalism Initiative', un acuerdo al que pueden adherirse las compañías a fin de fomentar la transparencia en la publicación de métricas sobre factores sociales y de gobernanza, entre otros. Si bien es cierto que en España todavía queda un largo camino por recorrer y numerosos desafíos, dadas las disparidades legislativas y culturales que pueden hacer la diferencia entre los países examinados, se hace un llamamiento a los responsables de política pública principalmente de los países latinoamericanos para centrar más esfuerzos en esta dirección.

Las compañías, agentes en el epicentro del cambio, deberían incluir en sus agendas más cuestiones sobre diversidad -en este caso, de género- por motivos, además de sociales, económicos como, por ejemplo, el mayor volumen de talento disponible, la mayor atracción y retención de talento femenino, las ventajas relacionadas con la reputación corporativa o el aumento del valor de su employer branding, todos ellos con poder explicativo de la rentabilidad y el éxito empresarial. Es por ello que se sugiere que todas las compañías (y en particular las que poseen plantillas y posiciones directivas con una alta representación masculina) reflexionen acerca de la necesidad, por un lado, de implantar programas o actuaciones que, siendo realistas, sean proactivas y contundentes $\mathrm{y}$, por otro, de medir el alcance de tales actuaciones y prácticas para poder evaluar la mejora y el progreso.

La sociedad en su conjunto cobra también uno de los papeles más relevantes a la hora de participar en un cambio de normas y estructuras para eliminar las incongruencias de roles y favorecer la igualdad. Es en este punto donde se critica el comportamiento que da lugar al síndrome de la abeja reina con la intención de que las mujeres sean las primeras protagonistas de la formación de alianzas y el cambio. Y un buen indicador de cambio sería que las cuotas de género dejaran de ser necesarias en el futuro más próximo.

Finalmente, las conclusiones obtenidas deben considerarse a la luz de una serie de limitaciones. La primera de ellas tiene que ver con la naturaleza de la información utilizada para el análisis, la cual puede estar sesgada en favor de los intereses de las propias compañías que las publican. La segunda hace referencia a la imposibilidad de establecer una relación causa-efecto y no conocer, según el caso, si las iniciativas que contribuyen a la promoción de mujeres son las que causan el incremento de la representación femenina en los gobiernos corporativos o, por el contrario, una mayor presencia femenina en posiciones de liderazgo es la que 'empuja' a un mayor número de acciones para el beneficio de ellas. Otra limitación se refiere a las diferencias que existen en cuanto al tamaño del universo empresarial en cada país, lo que dificulta la proporcionalidad en la comparativa. Es por esto que llevar a cabo un análisis que permita probar causalidad y profundizar en el impacto de los programas a través de fuentes de información primaria podría enriquecer las conclusiones de este trabajo. 


\section{Referencias bibliográficas}

Campos-García, I., Olivas-Luján, M., \& Zúñiga-Vicente, J.Á. (2019). Managing Gender Diversity in Companies Operating in Different National Contexts: The Case of Spanish MNCs in Latin American Countries. In Diversity within Diversity Management: Types of Diversity in Organizations (pp. 197-219). Emerald Publishing Limited.

https://doi.org/10.1108/S1877-636120190000022011

Catalyst (2020). Women in Management: Quick Take. Recuperado de: https://www.catalyst.org/research/ women-in-management/\#: :text=Globally $\% 20$ the $\% 20$ Share $\% 20$ of $\% 20$ Women $\% 20$ in $\% 20$ Senior $\% 20$ Management $\% 20$ is $\% 20$ Increasing $\% 20$ Incrementally\&text $=\mathrm{In} \% 202019 \% 2 \mathrm{C} \% 20$ the $\% 20$ proportion $\% 20$ of, this $\% 20$ percentage $\% 20$ remains $\% 20$ the $\% 20$ same.

CEPAL (2020). La autonomía de las mujeres en escenarios económicos cambiantes. Recuperado de: https://repositorio.cepal.org/bitstream/ handle/11362/45032/4/S1900723 es.pdf

Deloitte (2019). Women in the boardroom: A global perspective - 6th Edition. Recuperado de:

https://www2. deloitte.com/global/en/pages/risk/articles/ women-in-the-boardroom-global-perspective.html

Eagly, A. H., \& Carli, L. L. (2003). The female leadership advantage: An evaluation of the evidence. The Leadership Quarterly, 14(6), 807-834. DOI: 10.1016/j. leaqua.2003.09.004

Eagly, A. H., \& Wood, W. (2011). Social role theory. Handbook of Theories in Social Psychology, 2, 458-476.
Eriksson-Zetterquist, U., Styhre, A. (2008). Overcoming the glass barriers: reflection and action in the 'Women to the Top' programme. Gender, Work \& Organization, 15, 133-160. DOI:10.1111/j.1468-0432.2007.00366.x

Fortune (2020). The number of female CEOs in the Fortune 500 hits an all-time record. Recuperado de: https://fortune.com/2020/05/18/women-ceosfortune-500-2020/

Helgesen, S. (1995). The female advantage: Women's ways of leadership. Crown Business.

McKinsey (2018). Women in the workplace. Recuperado de: https://www.mckinsey.com/featured-insights/genderequality/women-in-the-workplace-2018

Olsen J.E., Parsons, C.K., Martins, L.L., Ivanaj, V. (2016). Gender diversity programs, perceived potential for advancement, and organizational attractiveness: An empirical examination of women in the United States and France. Group \& Organization Management, 41(3), 271-309. https://doi.org/10.1177/1059601115583579

Rosener, J. B. (2011). Ways women lead. In Leadership, Gender, and Organization (pp. 19-29). Springer, Dordrecht. https://doi.org/10.1007/978-90-481-9014-0_3

Swailes, S., Downs, Y., \& Orr, K. (2014). Conceptualising inclusive talent management: Potential, possibilities and practicalities. Human Resource Development International, 17(5), 529-544. https://doi.org/10.1080/13678868.2014.954188

The World Bank (2020). Labor force participation rate, female ( $\%$ of female population ages $15+$ ) (modeled ILO estimate). Recuperado de: https://data.worldbank.org/ indicator/sl.tlf.cact.fe.zs?most recent year desc=true 